\title{
Schwarzer Humor im medizinischen Alltag - akzeptabel oder nicht?
}

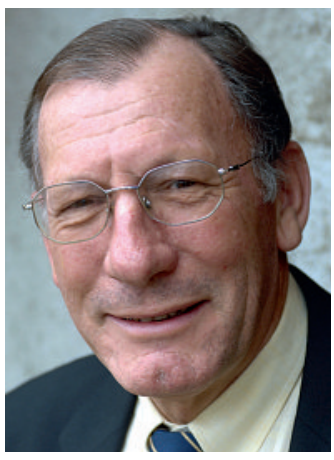

Jean Martin
«Humor ist, wenn uns die Wahrheit schneller und direkter gesagt wird, als wir dies gewohnt sind» (George Saunders). Was sollen wir davon halten, wenn Ärzte gelegentlich bestimmte Probleme ihrer Patienten mit schwarzem Humor kommentieren? NB: Wir sprechen hier nicht von schlüpfrigen Stammtischwitzen oder pejorativen Bemerkungen zu «schwierigen» Patienten, sondern von (nicht als Beleidigung gedachtem) Humor in Bezug auf den Zustand eines Kranken oder die Umstände seiner Versorgung, davon, was zwischen Fachleuten in Abwesenheit des Patienten - aber auch in seiner Anwesenheit - gesagt werden kann, um schwierige Situationen mit einem Lächeln zu meistern.

Katie Watson, Ethikerin und Teilzeit-Comedian, unterrichtet an der Northwestern University nahe Chicago und hat eine inhaltsreiche Studie zu diesem Thema veröffentlicht [1]. Zunächst muss der Bezug zwischen Macht und Humor geklärt werden. Man könnte meinen, dass es zulässig sei, über Einzelpersonen oder Personengruppen zu lachen, wenn sie wichtiger sind als wir - nicht jedoch im umgekehrten Fall. So können die Professoren bei der Examensfeier auf die Schippe genommen werden. Auch ein Lachen über den Chef kann hilfreich sein, will man den Konflikt mit ihm meiden.

Oft ist Witz eine Art Rebellion gegen eine - gegebenenfalls bedrückende - Allmacht und es gibt wenig Allmacht, die bedrückender ist als Tod, Krankheit oder Trauma. In den Konzentrationslagern kam es vor, dass die Gefangenen über ihre unmenschlichen Bedingungen lachten. Humor legt oft den Finger auf schmerzliche Absurditäten oder Ungereimtheiten und versucht, sie zu «integrieren». Es ist der Versuch, Sinn in eine sinnlose Welt zu bringen - was auch in klinischen Situationen vorkommt!

Möglicherweise ist der Beruf des Arztes so schwer, dass hinter den Kulissen alles erlaubt ist, vor allem, wenn Schwieriges bewältigt werden muss (coping). Damit lässt sich nicht alles entschuldigen, doch es besteht eine gewisse Relevanz. Schwarzer Humor ist ein Instrument, um mit dramatischen, bewegenden Situationen umzugehen, sie zu verdauen. Er hilft, wenn es darum geht, sich wieder zu fassen und seine Aufgabe bei anderen Patienten zu erfüllen. Katie Watson berichtet von jungen Ärzten in der Notaufnahme, die bei einem Pizzaservice eine Bestellung aufgegeben hatten. Der Pizzakurier wird unterwegs angeschossen ... Nach dem vergeblichen Versuch, ihn zu retten, und im Stress des Augenblicks fragt einer: «Was hätten wir ihm als Trinkgeld geben müssen?», und erntet einen grossen Lacher. Grenzwertig. Aber (Watson): «Wenn ich nach Unfall, Infarkt oder Vergewaltigung der nächste in der
Notaufnahme wäre, würde ich davon profitieren. Diese Ärzte brauchten den Witz, um weiterarbeiten zu können [...]. Schwarzer Humor kann auch als Respekt vor der bevorstehenden Arbeit gewertet werden.» Es ist eine Art, Abstand zu nehmen.

Watson zitiert eine erfahrene Psychiaterin, die von sich sagt, gelegentlich Witze in Bezug auf ihre Patienten zu machen, die jedoch meint, jüngere hätten sich noch nicht das Recht dazu erworben ... Eigentlich eine diskriminierende Bemerkung, die jedoch auch Sinn machen kann. Die (nicht aus Büchern zu lernende!) Erfahrung erlaubt eine bessere Einschätzung der Situation, eine Bewertung des Möglichen. Andere Aspekte sind zu berücksichtigen. Junge Mediziner (oder Studenten im Seziersaal) verwenden häufiger als ihre erfahrenen Kolleginnen und Kollegen schwarzen Humor, um Spannung abzubauen (in anderen Fällen kann es - bei verachtenden Bemerkungen zu Kranken - aber auch genau umgekehrt sein, dies ist jedoch nicht Thema des Aufsatzes). Zu Beginn ihrer Laufbahn sind junge Ärzte und Pflegende häufiger in direktem Kontakt zu den Sorgen und Nöten, der Frustration und manchmal der Wut von Patienten und ihren Familien und damit häufiger moralisch gefordert.

Gelegentlich könnte man sagen, dass eher über die Situation und nicht wirklich über den Kranken gewitzelt wird. Nicht unbedingt eine gute Ausrede - und wie liesse sich da ein Unterschied aufzeigen? Also schwarzer Humor in der Pflege? Möglicherweise ja, aber differenziert. Der «therapeutische Humor» zwischen Pflegenden und Patienten sollte nicht ausgeschlossen werden. Er entspannt die Situation und macht deutlich, dass gemeinsam gekämpft wird und wir alle es mit einer sehr unvollkommenen condicio humana zu tun haben. Dazu Katie Watson: «Die Medizin ist eine seltsame Wissenschaft, in der von normalen Menschen verlangt wird, so zu tun, als würden Kot oder Erbrochenes nicht riechen, als gäbe es keine veränderten Körper und als sei der Tod nicht erschreckend» ...

Bleibt die Frage, ab wann der schwarze Humor zu einer Art Missbrauch wird? Watson: Wenn wir ihn nicht ganz aus der Medizin verbannen wollen, bewegen wir uns jedoch auf glattem Terrain («We are on thin ice»). Pflegende müssen offen und taktvoll agieren, mit Bedacht vorgehen, inklusive und vor allem wenn sie Witze machen. Denn - so Nell Kirst [2] in einem Artikel derselben Fachzeitschrift - dies ist der «Preis, den wir dafür zahlen müssen, dass uns die Patienten erlauben, in ihre Privatsphäre einzudringen». 